\title{
ANÁLISIS DE LA REFLEXIÓN REALIZADA POR UN FUTURO PROFESOR SOBRE EL PAPEL DE LA MODELIZACIÓN MATEMÁTICA EN LA MEJORA DE UN PROCESO DE INSTRUCCIÓN PARA ENSEÑAR TRIGONOMETRÍA
}

\author{
Carlos Ledezma \\ cledezar25@alumnes.ub.edu \\ https://orcid.org/0000-0001-9274-7619 \\ Universitat de Barcelona (UB) \\ Barcelona, España \\ Vicenç Font \\ vfont@,ub.edu \\ https://orcid.org/0000-0003-1405-0458 \\ Universitat de Barcelona (UB) \\ Barcelona, España \\ Gemma Sala \\ gsala@ub.edu \\ https://orcid.org/0000-0001-9830-312X \\ Universitat de Barcelona (UB) \\ Barcelona, España
}

Recibido: 22/10/2020 Aceptado: 09/02/2021

\begin{abstract}
Resumen
El objetivo de este estudio es analizar la reflexión realizada por un futuro profesor (estudiante de un máster de formación inicial de profesores de matemáticas de secundaria) sobre el papel de la modelización matemática en la mejora de un proceso de instrucción para enseñar trigonometría. En particular, después de explicar el contexto institucional de formación y de cómo se desarrolla la reflexión sobre la propia práctica en este máster, nos centraremos en un estudio de caso de un profesor analizando la reflexión que hace en su Trabajo Final de Máster, donde valora la implementación de una secuencia de tareas en su periodo de prácticas para hacer una propuesta de rediseño orientada a su mejora. El interés se centra en estudiar el uso que hace el futuro docente de los criterios de idoneidad didáctica (propuestos por el Enfoque OntoSemiótico) en la memoria escrita de su trabajo final de máster. La metodología consiste en realizar un análisis de contenido de la reflexión realizada en esta memoria escrita y, desde el punto de vista de la extensión, se trata de un estudio de caso. Un primer resultado es que, de la reflexión y de las tareas que propone, se infiere que el futuro profesor no diferencia claramente entre los procesos 'resolución de problemas', 'contextualización' y 'modelización'. Un segundo resultado es que este profesor asocia la incorporación de tareas para desarrollar el proceso de modelización con la mejora sobre todo de las idoneidades epistémica y ecológica del proceso de instrucción implementado.
\end{abstract}

Palabras clave: Criterios de idoneidad didáctica. Formación inicial de profesores. Modelización matemática. Trabajo final de máster. Trigonometría.

ANÁLISE DA REFLEXÃO REALIZADA POR UM FUTURO PROFESSOR SOBRE O PAPEL DA MODELAGEM MATEMÁTICA NA MELHORIA DE UM PROCESSO DE INSTRUÇÃO PARA O ENSINO DE TRIGONOMETRIA 


\title{
Resumo
}

O objetivo deste estudo é analisar a reflexão feita por um futuro professor (aluno de um curso de mestrado de formação inicial de professores de matemática do ensino secundário na Espanha) sobre o papel da modelagem matemática no aprimoramento de um processo de instrução para o ensino de trigonometria. Em particular, depois de explicar o contexto institucional de formação e o modo como se desenvolve a reflexão sobre a própria prática nesse mestrado, focaremos no estudo de caso de um professor, analisando sua reflexão desenvolvida na sua Dissertação de Mestrado, onde avalia uma sequência de tarefas, implementadas no seu período de estágio, para fazer uma proposta de redesenho visando o aprimoramento dessas atividades. O interesse central deste estudo é verificar o uso que o futuro professor faz dos critérios de idoneidade didática (propostos pelo Enfoque Onto-Semiótico) evidenciado no seu trabalho de conclusão de curso. A metodologia consiste na realização de uma análise de conteúdo da reflexão feita na dissertação de mestrado e, do ponto de vista da extensão, é um estudo de caso. Um primeiro resultado é que, da reflexão e das tarefas que propõe, infere-se que o futuro professor não faz distinção clara entre os processos 'resolução de problemas', 'contextualização' e 'modelagem matemática'. Um segundo resultado é que esse professor associa a incorporação de tarefas para desenvolver o processo de modelagem com o aperfeiçoamento, sobretudo, das idoneidades epistêmica e ecológica do processo de instrução implementado.

Palavras chave: Critérios de idoneidade didática. Formação inicial de professores. Modelagem matemática. Dissertação de mestrado. Trigonometria.

\section{ANALYSIS OF A FUTURE TEACHER'S REFLECTION ON THE ROLE OF MATHEMATICAL MODELLING FOR IMPROVING AN INSTRUCTIONAL PROCESS FOR THE TEACHING OF TRIGONOMETRY}

\begin{abstract}
The aim of this study is to analyse the reflection made by a future teacher (a student of a master's degree in initial secondary school mathematics teachers' training) on the role of mathematical modelling for improving an instructional process for the teaching of trigonometry. Particularly, after explaining the institutional context of training and how the reflection on the own practice is made in this master program, we will focus on a case study of a teacher analysing the reflection that he makes in his Master's Thesis, where he values the implementation of a sequence of tasks in his internship period to make a redesign proposal aimed at its improvement. The interest of this work focuses on how the future teacher uses the didactic suitability criteria (proposed by the Onto-Semiotic Approach) in the written report of his master's thesis. The methodology consists of carrying out a content analysis made in his written report and, from the point of view of its extension, it is a case study. The first result is that, based on the reflection and the tasks that he proposes, it is interred that the future teacher does not clearly differentiate between the processes 'problem solving', 'contextualisation', and 'modelling'. The second result is that this teacher relates the addition of tasks to develop the modelling process with the improvement of the epistemic and ecological suitability of the implemented instructional process.

Keywords: Didactic suitability criteria. Initial teachers' training. Master's thesis. Mathematical modelling. Trigonometry.
\end{abstract}




\section{Introducción}

Un aspecto clave en la formación del profesorado es la reflexión docente sobre su propia práctica, pues el profesor es considerado, en términos de Schön (1983, 1987), como un sujeto reflexivo y racional capaz de formular opiniones, tomar decisiones, tener creencias y generar su propia rutina de desarrollo profesional; al mismo tiempo que este tipo de pensamientos, influyen en su comportamiento e, inclusive, le definen. En esta misma línea, Schoenfeld y Kilpatrick (2008) plantean que, una vez que la reflexión se hace algo habitual en el profesor, se puede convertir en el principal mecanismo para la mejora de su propia práctica educativa.

Diversos estudios han abordado el tema de la reflexión docente en los procesos de formación del profesorado de matemáticas, específicamente, utilizando como herramienta los criterios de idoneidad didáctica que propone el Enfoque Onto-Semiótico (véase, Breda, 2020; Breda, Font, Lima y Villela, 2018; Breda y Lima, 2016; Breda, Pino-Fan y Font, 2017; Font, Breda, Seckel y Pino-Fan, 2018; Pino-Fan, Godino y Font, 2016, entre otros), en los cuales se analizan los Trabajos Finales de Máster (TFM) de los futuros profesores. Si bien estos estudios se han interesado por analizar la reflexión docente en su totalidad, el que se reporta en este artículo centra su interés en la reflexión docente sobre la implementación de la modelización matemática en la mejora de un proceso de instrucción.

A partir de esto último, uno de los interrogantes que emerge en este estudio es: ¿cómo utilizan los profesores en formación los criterios de idoneidad didáctica en sus trabajos finales de máster para reflexionar sobre la implementación de la modelización? Para responderla, en este artículo se analiza la reflexión realizada por un futuro docente (Albiol, 2020) sobre el papel de la modelización matemática en la mejora de un proceso de instrucción, para la cual hizo uso de los criterios de idoneidad didáctica. En términos generales, el TFM escogido corresponde a una reflexión y reformulación de la unidad didáctica que diseñó e implementó este docente en formación, en la que se contempló el trabajo con modelización matemática para la enseñanza de la trigonometría en el cuarto curso de Enseñanza Secundaria Obligatoria (estudiantes de 15 a 16 años).

La estructura de este artículo es la siguiente: a continuación de la introducción, en el segundo apartado, se presentan los referentes teóricos considerados en este estudio, a saber, una caracterización del proceso y de un problema de modelización, y la noción de idoneidad didáctica propuesta por el Enfoque Onto-Semiótico (EOS); en el tercer apartado se explica la 
metodología utilizada en este estudio que, básicamente, consiste en realizar un análisis de contenido; en el cuarto apartado se presenta el análisis de la reflexión que hizo el autor del TFM sobre su unidad didáctica y de los problemas de modelización que propuso; finalmente, se discuten los análisis y se presentan algunas consideraciones finales.

\section{Marco teórico}

En este apartado se presentan los referentes teóricos considerados en este estudio.

\subsection{Modelización matemática}

El proceso de modelización es entendido, en términos generales, como un pasaje sistémico entre el mundo real y el matemático, para así dar solución a una situación-problema de la realidad. Si bien se han diseñado diferentes ciclos para explicar este proceso (Borromeo Ferri, 2006), y han emergido distintas perspectivas sobre su implementación (Abassian, Safi, Bush y Bostic, 2020), existe un claro consenso en que su inclusión curricular es necesaria para mejorar el aprendizaje de la matemática. Sobre esto último, Blum (2011) menciona que el trabajo con modelización matemática es beneficioso para los estudiantes, ya que favorece la comprensión del mundo; provee de un soporte para la motivación, y la formación, comprensión y retención de conceptos; contribuye al desarrollo de distintas competencias matemáticas asociadas; y ayuda a mejorar la imagen que se tiene de la matemática.

Conjuntamente se encuentran las aplicaciones matemáticas, pues ambos procesos, los referidos a las aplicaciones y la modelización, denotan todo tipo de relaciones que se puedan establecer entre el mundo real y el matemático. La diferencia entre ambos radica, en términos de Blum (1994, 2002), en que la modelización se produce desde la realidad hacia la matemática, enfatizando en el aspecto procedimental de dicha transición; y las aplicaciones se producen en el sentido contrario, enfatizando en los objetos matemáticos involucrados en ésta.

En este estudio no se adopta un ciclo o perspectiva de modelización en particular, sino más bien, se consideran algunos atributos consensuados que caracterizan a un problema de modelización matemática como tal. Es por ello que se adopta la propuesta de Maaß (2010), en la que se destacan seis cualidades de este tipo de problemas y, a modo de ejemplo, se utiliza el enunciado de la Figura 1 para ejemplificar estas características, en la línea de lo planteado por Borromeo Ferri (2018). 
Figura 1. Ejemplo de problema de modelización

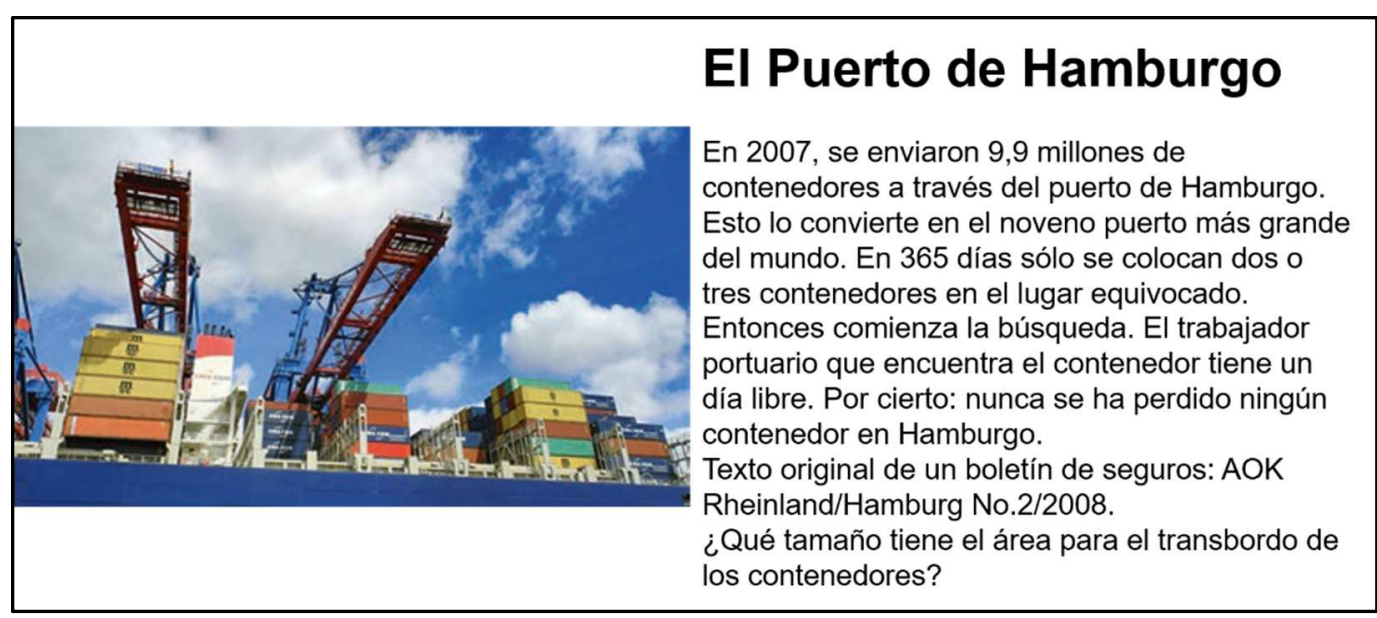

Fuente: Traducido desde Borromeo Ferri (2018, p. 44)

El problema 'Puerto de Hamburgo' se caracteriza por ser abierto, y no estar limitado a una respuesta o procedimiento específicos; además, la tarea es compleja ya que, dada la información que aporta el enunciado, los estudiantes deben buscar los datos que sean relevantes. El contexto en que está situado el enunciado lo hace ser realista, pues incorpora elementos del mundo real (los contenedores, el puerto, la ciudad de Hamburgo); de la mano de esta característica se encuentra la de autenticidad, ya que no se presenta a los estudiantes una pseudorealidad, sino que la situación descrita es coherente con un hecho que ha ocurrido o que pueda ocurrir en la realidad (Palm, 2007). Otra característica es que un problema de modelización matemática es, en esencia, un problema que no puede ser resuelto a través de la aplicación de algoritmos conocidos (Schoenfeld, 1994), pero que sí requiere de estrategias para su resolución, y cuya respuesta no puede ser hallada siguiendo procedimientos rutinarios (Lesh y Doerr, 2003). Finalmente, un problema de modelización debiese ser solucionable a través del ciclo de modelización, lo que implica que para su resolución se utilicen todas las fases que lo componen (Borromeo Ferri, 2018).

Las seis características antes descritas se asocian con lo que, en términos de Julie y Mudaly (2007) y de Guerrero-Ortiz y Mena-Lorca (2015), se considera como la perspectiva 'modelización como un contenido', en la cual el objetivo es desarrollar la habilidad de modelizar, y se logra por medio de la construcción de modelos matemáticos que emergen de los conocimientos adquiridos por los estudiantes. La otra perspectiva que plantean estos autores es la de 'modelización como un vehículo', en la que este proceso se utiliza para desarrollar la 
comprensión matemática, por medio de la construcción de conocimientos matemáticos a partir de la modelización.

\subsection{Criterios de idoneidad didáctica}

En el EOS se entiende la idoneidad didáctica de un proceso de enseñanza-aprendizaje como el grado en que éste (o una parte del mismo) reúne ciertas características que permiten calificarlo como idóneo (óptimo o adecuado) para conseguir la adaptación entre los significados personales logrados por los estudiantes (aprendizaje) y los significados institucionales pretendidos o implementados (enseñanza), teniendo en cuenta las circunstancias y recursos disponibles (entorno). Se trata de un constructo multidimensional que se descompone en idoneidades parciales (idoneidad epistémica, cognitiva, interaccional, afectiva, mediacional, y ecológica), componentes e indicadores. La operatividad de los criterios de idoneidad didáctica exige definir un conjunto de indicadores observables, que permitan valorar el grado de idoneidad de cada una de las facetas del proceso de instrucción (Breda y Lima, 2016). En el Cuadro 1 se presentan los criterios de idoneidad didáctica con su respectiva descripción.

Cuadro 1.

Criterios de idoneidad didáctica

\begin{tabular}{|l|l|}
\hline \multicolumn{1}{|c|}{ Criterios } & \multicolumn{1}{c|}{ Descripción } \\
\hline Epistémico & $\begin{array}{l}\text { Para valorar si las matemáticas que se enseñan son unas 'buenas } \\
\text { matemáticas'. }\end{array}$ \\
\hline Cognitivo & $\begin{array}{l}\text { Para valorar, antes de iniciar el proceso de instrucción, si lo que } \\
\text { se quiere enseñar está a una distancia razonable de lo que saben } \\
\text { los alumnos. }\end{array}$ \\
\hline Interaccional & $\begin{array}{l}\text { Para valorar si la interacción ha resuelto dudas y dificultades de } \\
\text { los alumnos. }\end{array}$ \\
\hline Mediacional & $\begin{array}{l}\text { Para valorar la adecuación de recursos materiales y temporales } \\
\text { utilizados en el proceso de instrucción. }\end{array}$ \\
\hline Afectivo (o emocional) & $\begin{array}{l}\text { Para valorar la implicación (interés, motivación) de los alumnos } \\
\text { en el proceso de instrucción. }\end{array}$ \\
\hline Ecológico & $\begin{array}{l}\text { Para valorar la adecuación del proceso de instrucción al proyecto } \\
\text { educativo del centro, las directrices curriculares, las condiciones } \\
\text { del entorno social y profesional, etc. }\end{array}$ \\
\hline
\end{tabular}

Fuente: Extraído desde Morales-López y Font (2019, p. 5)

En el EOS, el proceso de modelización es entendido como un híper o mega proceso (Godino, Batanero y Font, 2007, 2019), puesto que implica otros procesos más elementales, como los de representación, argumentación, idealización, generalización, etc. No obstante, en 
este marco se considera que potenciar la modelización matemática es un aspecto que mejora la idoneidad del proceso de instrucción (Sala, Font, Giménez y Barquero, 2017).

\section{Metodología}

Para dar cumplimiento al objetivo propuesto, se siguió una metodología de investigación cualitativa (Cohen, Manion y Morrison, 2018), que consistió en realizar un análisis de contenido de la reflexión realizada por un futuro profesor en su TFM. Por otro lado, desde el punto de vista de la extensión, se puede afirmar que se trata de un estudio de caso (Stake, 2005). En este apartado, primero se presenta el contexto en que se desarrolló este estudio, para luego describir la organización del TFM que se analizó. Finalmente, se detallan los aspectos metodológicos empleados para el análisis de contenido.

\subsection{Contexto de la investigación}

Esta investigación se desarrolló en el contexto del Máster de Formación del Profesorado de Educación Secundaria Obligatoria y Bachillerato (especialidad de matemáticas), impartido por las universidades de Catalunya (España), durante el curso 2019-2020.

En las Facultades de Matemáticas de España no se contempla un itinerario cuya salida profesional sea conducente al título de Profesor de Matemáticas de Secundaria, sino que se sigue un modelo llamado -metafóricamente- de formación inicial en paralelo. De este modo, para ser profesor de matemáticas se sigue un proceso que consiste, primero, en una formación disciplinar y, a continuación, en una formación profesionalizante. Después de los estudios de grado disciplinares, los futuros profesores deben cursar un máster de 60 créditos ETCS (sistema europeo de transferencia y acumulación de créditos vigente en el Espacio Europeo de Educación Superior) organizado por competencias. Los créditos se organizan en torno a tres módulos, subdivididos a su vez en materias o asignaturas:

- Módulo genérico: Aprendizaje y desarrollo de la personalidad; Procesos y contextos educativos; Sociedad, familia y educación.

- Módulo específico: Complementos para la formación disciplinar; Aprendizaje y enseñanza de las materias correspondientes; Innovación docente e iniciación a la investigación educativa.

- Módulo de prácticas: Prácticas en centros de secundaria. Realización del TFM.

Las directrices del Máster prescriben la realización del prácticum, en colaboración con las instituciones educativas establecidas mediante convenios entre universidades y 
administraciones educativas. Estas últimas, deben estar reconocidas como centros de prácticas, así como los tutores encargados de la orientación y tutela de los estudiantes.

Las competencias profesionales de este Máster se estructuran en tres tipos: genéricas, específicas (en este caso, de las matemáticas y su didáctica), y las que se desarrollan por medio de la práctica. A modo de ejemplo, las siguientes competencias son algunas de las desarrolladas en este programa de formación:

- Competencia genérica: Participar en la definición del proyecto educativo y en las actividades generales del centro, atendiendo a criterios de mejora de la calidad, atención a la diversidad, prevención de problemas de aprendizaje y convivencia.

- Competencia específica: Identificar los problemas relativos a la enseñanza y aprendizaje de las matemáticas, y plantear alternativas y soluciones.

- Competencia relacionada con la práctica: Participar en las propuestas de mejora en los distintos ámbitos de actuación a partir de la reflexión basada en la práctica.

En su programa de estudios se incluye, dentro del módulo específico, un submódulo sobre modelización matemática, en el que se presenta a los profesores en formación el ciclo propuesto por Blum y Leiß (2007), junto con distintos ejemplos en que se utiliza, y se les solicita que planteen un problema de modelización (enunciado, resolución, ubicación curricular de los contenidos) como trabajo final del submódulo. Para la obtención del grado, los futuros docentes deben diseñar una secuencia de enseñanza-aprendizaje que deben implementar en el módulo de prácticas, que está determinada por la institución educativa, el nivel de los estudiantes, y el momento del año escolar en que realicen su intervención. Dada esta situación, el margen que tiene el docente en formación para introducir la modelización en la implementación de su unidad didáctica, está sometido a muchas restricciones, no así en el rediseño propuesto en su TFM.

\subsection{Descripción del TFM}

En las orientaciones generales que se dan a los estudiantes del máster para la elaboración del TFM, se les dice que debe ser un trabajo original, autónomo e individual, que permita al estudiante mostrar de forma integrada los contenidos formativos recibidos y las competencias generales asociadas al título de Máster en Formación del Profesorado de Secundaria de Matemáticas, y que debe contribuir a reflexionar y profundizar en el análisis de su propia práctica, posibilitando proponer elementos de mejora de la misma. El TFM es más que una simple reflexión escrita de la práctica escolar realizada, sin embargo, el nivel de habilidades de 
investigación requeridas es menor que en una tesis de máster orientada a la investigación. Aunque se alienta a los profesores en formación a justificar la mejora de su secuencia de aprendizaje con los resultados de la comunidad de investigadores en Didáctica de la Matemática sobre el tema desarrollado en su práctica, en general, se citan pocas referencias en estos trabajos.

Un elemento clave del TFM es su relación directa con la experiencia escolar realizada previamente, es decir, con el diseño y la implementación de una unidad didáctica. Para guiar todo este trabajo, se asigna un mismo tutor al docente en formación durante el periodo de prácticas (donde se ha diseñado e implementado la unidad didáctica) y durante el de elaboración del TFM, para facilitar así tanto la continuidad de las prácticas y del proceso de reflexión sobre éstas, como para poder reconocer los progresos alcanzados. Durante este periodo se realizan, como mínimo, cuatro reuniones entre el estudiante y su tutor: dos durante su práctica escolar, y otras dos durante la realización de la memoria escrita.

Después de la práctica educativa, se presentan a los futuros profesores los criterios de idoneidad didáctica propuestos por el EOS, junto con la versión modificada de la pauta de componentes y descriptores de dichos criterios que permite aplicarlos (Godino, 2013). Con estas herramientas, se les sugiere que valoren en su TFM la secuencia de aprendizaje que implementaron para que, de este modo, propongan cambios que puedan ayudar a mejorar la idoneidad del proceso de instrucción. En su análisis, se les sugiere que consideren responder a preguntas como las siguientes: a) ¿he enseñado unas matemáticas de calidad?, ¿se puede mejorar esta calidad?, ¿cómo?; b) ¿se podían lograr aprendizajes con las actividades propuestas?, ¿han aprendido los alumnos?, ¿por qué no?; c) ¿se podría mejorar la gestión de la clase?; d) ¿usé los recursos adecuados?, ¿el tiempo estuvo bien gestionado?; e) ¿cómo se ha considerado la perspectiva ecológica en las condiciones generales del trabajo?

El trabajo de Albiol (2020) consiste en una propuesta para abordar la enseñanza de la trigonometría en el cuarto curso de ESO, a partir de los teoremas de Pitágoras y Thales. El documento se estructura en cuatro secciones temáticas: en la introducción general se presenta el contexto en que se implementó la unidad didáctica (centro educativo, alumnado, adecuación curricular de la unidad didáctica); en el análisis y valoración, el autor explica la valoración que hace de los seis criterios de idoneidad didáctica con respecto a su unidad didáctica (reflexión sobre la práctica); en la propuesta de mejora se detallan los aspectos de la unidad didáctica que se proponen mejorar para futuras implementaciones, lo que incluye el rediseño de las tareas 
(reformulación de la unidad didáctica); y en las consideraciones finales, el autor concluye sobre su trabajo y realiza una valoración de sus aprendizajes en el máster.

El objetivo de aprendizaje de la unidad diseñada es que "los alumnos sean capaces de emplear conocimientos geométricos y de trigonometría para la resolución de problemas" (Albiol, 2020, p. 5, traducción propia), el cual se ha planteado a partir de las competencias dispuestas en el currículo (Departament d'Educació, 2019). La propuesta constaba de un total de 14 sesiones, de las cuales seis se desarrollaron de manera presencial y las ocho restantes de manera telemática (online), ello producto del confinamiento de la población a causa de la pandemia por COVID-19. El autor enfoca su propuesta en un sentido competencial, es decir, dotando a los contenidos de una utilidad, a fin de que los estudiantes los puedan valorar como una herramienta, y que desarrollen la capacidad de utilizarlos en diversos contextos (Albiol, 2020).

\subsection{Análisis de contenido}

En su TFM, el profesor en formación escribe comentarios de tipo valorativo, relacionados con los diferentes componentes e indicadores de los criterios de idoneidad didáctica. Si bien esta valoración ha sido triangulada en una primera instancia con el tutor, en la presentación oral ante el tribunal del TFM hay una segunda triangulación. Para esta investigación, el foco se centró en el análisis de las valoraciones que hizo el futuro docente sobre el trabajo con modelización matemática. Para el análisis del contenido, se parte de categorías apriorísticas, a saber, los criterios, componentes y descriptores de idoneidad didáctica, y para su operacionalización, se siguió una metodología similar a la empleada por Breda et al. (2017).

El análisis cualitativo de los datos se desarrolló en cuatro pasos. Primero, se clasificaron los 122 TFMs (correspondientes al curso 2019-2020) de acuerdo con el nivel educativo $\left(1^{\circ}\right.$ a $4^{\circ}$ de ESO, $1^{\circ}$ y $2^{\circ}$ de bachillerato) y al contenido matemático (geometría, trigonometría, álgebra, etc.) que abordaron en sus propuestas didácticas. Segundo, se realizó una búsqueda de palabras clave afines a la modelización (context*, model*, problema*, real*, etc.), para así seleccionar aquéllos que abordaron esta temática. Tercero, se categorizaron los TFMs de acuerdo con la importancia que le dieron sus autores a la modelización en su unidad didáctica, es decir, si la consideraron en sus implementaciones, en su propuesta de rediseño, o en ambas. Finalmente, se analizaron las valoraciones de las idoneidades didácticas para identificar en qué criterios privilegiaron sus reflexiones sobre modelización. 
Se escogió esta memoria escrita en particular, pues es una en las que explícitamente se hace alusión a la incorporación de la modelización, tanto en la unidad didáctica implementada como en la propuesta de rediseño orientada a su mejora.

\section{Análisis del TFM}

En este apartado se presenta el análisis de contenido del TFM. Para ello, primero se presentan las valoraciones que hace el docente en formación sobre la implementación de su unidad didáctica por medio de la utilización de los criterios de idoneidad didáctica, además de destacar tanto los comentarios con los que justifica su reflexión y que aluden -directa o indirectamente- a la modelización, como los problemas que propone para este proceso. Seguidamente, se presenta la valoración global que hace el autor del TFM sobre el proceso de instrucción implementado y, finalmente, se describe el rediseño para la mejora de su unidad didáctica.

\subsection{Idoneidad epistémica}

La valoración de la idoneidad epistémica que realiza el futuro profesor Albiol (2020) sobre su unidad didáctica, da como resultado 3,7 sobre $5(3,7 / 5)$ a partir de la media de las siguientes puntuaciones por componente: errores y ambigüedades (5), riqueza de procesos (4), representatividad de la complejidad (2). El profesor en formación considera en forma conjunta los componentes 'errores' y 'ambigüedades', y los valora con la puntuación máxima aduciendo a que los contenidos fueron revisados con anterioridad para que fuesen concretos y contextualizados, a fin de evitar errores.

En el componente 'riqueza de procesos', el futuro docente señala que ha trabajado los procesos de comunicación, formulación, argumentación, institucionalización, resolución de problemas, tratamiento y conversión, y contextualización (modelización). Sobre el proceso 'resolución de problemas', Albiol (2020) define que es "la capacidad de un alumno de resolver diferentes tipos de problemas matemáticos con diversos métodos” (p. 9, traducción propia); sobre el proceso 'contextualización (modelización)', define que es el "uso de problemas del mundo real” (p. 9, traducción propia). Con respecto a este último, específicamente, plantea los problemas presentados en la Figura 2 y Figura 3: 
Figura 2. Primer problema de contextualización (modelización) propuesto en el TFM

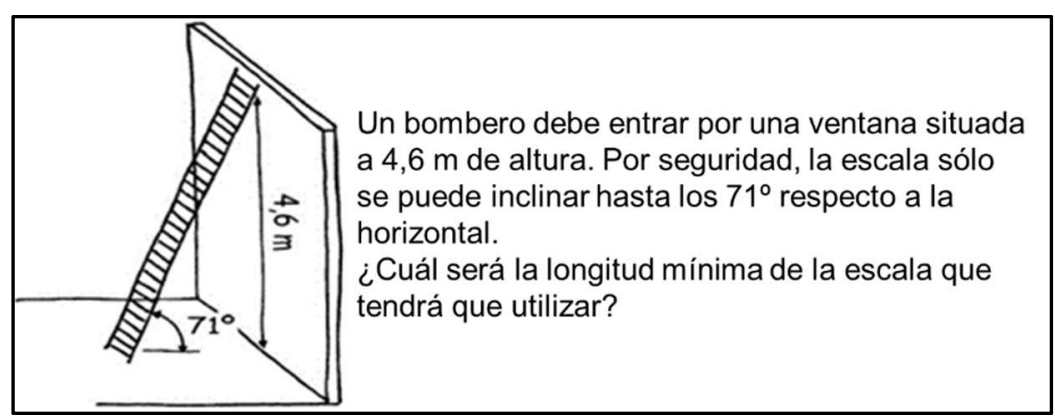

Fuente: Traducido desde Albiol (2020, p. 126)

Figura 3. Segundo problema de contextualización (modelización) propuesto en el TFM

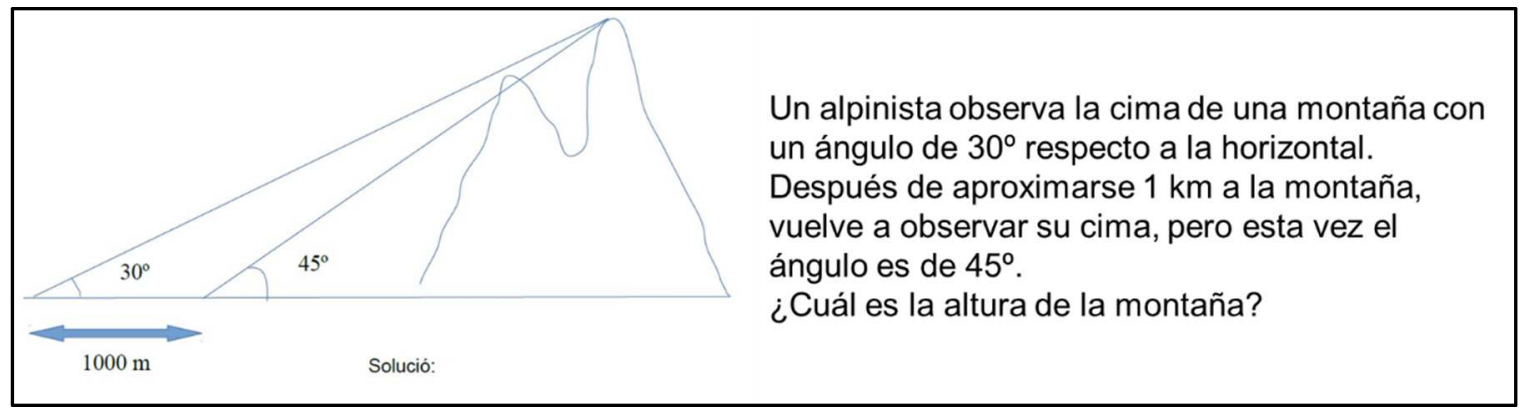

Fuente: Traducido desde Albiol (2020, p. 128)

En términos generales, el docente en formación considera que los procesos incluidos en su unidad didáctica (comunicación, formulación, argumentación, institucionalización, resolución de problemas, tratamiento y conversión, contextualización [modelización]) fueron bien trabajados, aunque cree necesario incluir también los de 'exploración' y 'algoritmización'.

Finalmente, en el componente 'representatividad de la complejidad', el autor del TFM señala que "los significados parciales son una muestra representativa de la complejidad matemática que se ha querido enseñar" (Albiol, 2020, p. 10, traducción propia), destacando el uso coherente que le ha dado a estos significados para describir la complejidad de los objetos matemáticos, y justificando esta valoración en términos de lo descrito por Font, Godino y Gallardo (2013) desde el punto de vista pragmatista. En contraposición con lo anterior, el autor asigna una baja puntuación a esta componente al considerar que "la representatividad de la complejidad [...] a lo largo de la unidad didáctica ha sido algo escasa" (Albiol, 2020, p. 10, traducción propia), ante lo cual analiza la representatividad de los significados parciales: teorema de Thales, teorema de Pitágoras, y razones trigonométricas (seno, coseno y tangente). 


\subsection{Idoneidad cognitiva}

La valoración de la idoneidad cognitiva que realiza el futuro profesor Albiol (2020) sobre su unidad didáctica, da como resultado 3,75 sobre $5(3,75 / 5)$ a partir de la media de las siguientes puntuaciones por componente: conocimientos previos (3), adaptación curricular a las diferencias individuales (5), aprendizaje (4), alta demanda cognitiva (3). En el componente 'conocimientos previos', debido a que los estudiantes evidenciaron (en la evaluación inicial) dificultades conceptuales y procedimentales con los teoremas de Pitágoras y Thales, el profesor en formación destaca el uso de ejercicios contextualizados para repasar dichos contenidos. Sin embargo, no especifica a cuáles se refiere concretamente cuando realiza esta valoración.

En los componentes 'adaptación curricular a las diferencias individuales' y 'aprendizaje', el futuro docente no hace alusiones de ningún tipo a los procesos 'resolución de problemas' o 'contextualización (modelización)'. En el componente 'alta demanda cognitiva', el docente en formación menciona la reformulación del problema 'Pendiente Coronavirus' (ver Figura 4), al cual se refiere en mayor detalle dentro del criterio de idoneidad ecológica.

\subsection{Idoneidad interaccional}

La valoración de la idoneidad interaccional que realiza el futuro profesor Albiol (2020) sobre su unidad didáctica, da como resultado 4 sobre $5(4 / 5)$ a partir de la media de las siguientes puntuaciones por componente: interacción docente-discente (4), interacción entre discentes (4), autonomía (4), evaluación formativa (4). Si bien, en ninguno de los cuatro componentes de este criterio, el autor del TFM se refiere directamente a los problemas de contextualización (modelización) propuestos a sus estudiantes, sí destaca el rol del trabajo colaborativo entre éstos para la resolución de la mayoría de las tareas.

\subsection{Idoneidad mediacional}

La valoración de la idoneidad mediacional que realiza el futuro docente Albiol (2020) sobre su unidad didáctica, da como resultado 4,3 sobre $5(4,3 / 5)$ a partir de la media de las siguientes puntuaciones por componente: recursos materiales (5), número de alumnos, horario y condiciones del aula (5), tiempo (3). En el componente 'recursos materiales', el profesor en formación destaca la disponibilidad de recursos TIC con la que contó, tanto durante la fase de implementación presencial (aulas con proyector, pizarra digital, ordenador fijo, tabletas para cada alumno, material manipulativo), como en la de trabajo online (acceso completo a la suite de Google), lo cual favoreció el trabajo con sus estudiantes para la resolución de ejercicios y 
problemas propuestos en su unidad didáctica. Sin embargo, en el componente 'tiempo', hace alusión a ciertas dificultades que presentó con sus estudiantes durante el periodo de trabajo online al abordar estas actividades. Al igual que en el criterio anterior, no se hacen valoraciones explícitas sobre los problemas propuestos.

Debido al periodo de trabajo online, el docente en formación declara lo siguiente:

Se hizo uso de plataformas para hacer ejercicios y problemas, como ThatQuiz y Socrative, clases y reuniones online por MeetGoogle. Sin embargo, había algunos alumnos que no conectaban la cámara, y tampoco participaban en el chat. En este caso, la idoneidad mediacional no es óptima porque la comunicación entre el profesor y el alumno no está presente. El docente no sabe si realmente el alumno está activo y presente en la clase online. (Albiol, 2020, p. 21).

\subsection{Idoneidad emocional}

La valoración de la idoneidad emocional que realiza el futuro profesor Albiol (2020) sobre su unidad didáctica, da como resultado 4 sobre 5 (4/5) a partir de la media de las siguientes puntuaciones por componente: intereses y necesidades (4), actitudes (4), emociones (4). En el componente 'intereses y necesidades', el profesor en formación considera un aspecto positivo el haber presentado ejemplos y aplicaciones realistas sobre los contenidos a enseñar en su unidad didáctica. No obstante, no hace alusión a los procesos de 'resolución de problemas' o 'contextualización (modelización)' en los dos componentes restantes de este criterio de idoneidad.

\subsection{Idoneidad ecológica}

La valoración de la idoneidad ecológica que realiza el futuro docente Albiol (2020) sobre su unidad didáctica, da como resultado 4 sobre 5 (4/5) a partir de la media de las siguientes puntuaciones por componente: adaptación al currículo (4), conexiones intra e interdisciplinares (4), utilidad socio-laboral (4), innovación didáctica (4). En el componente 'adaptación al currículo', el docente en formación considera que, tanto los contenidos abordados como los objetivos planteados, se encuentran respectivamente en consonancia con lo estipulado en los bloques temáticos 'Espacio y forma' y 'Medida', y con los criterios de evaluación que define el currículo para el cuarto curso de ESO (Departament d'Educació, 2019). 
En el componente 'conexiones intra e interdisciplinares', el futuro profesor señala que, en el ámbito intradisciplinar, ha realizado conexiones entre contenidos matemáticos, mientras que, en el interdisciplinar, las ha realizado con la historia (al hablar de Thales, Eratóstenes y Aristarco), la física (al trabajar con la Ley de Snell y la descomposición vectorial de fuerzas), y con el uso de recursos tecnológicos (al utilizar la planilla Excel y la aplicación GeoGebra). Sobre la conexión con la física, el profesor en formación declara que "la asignatura de matemáticas se convierte en una de las más importantes para la evaluación de competencias transversales" (Albiol, 2020, p. 22, traducción propia).

En el componente 'utilidad socio-laboral', el futuro docente destaca el hecho de que "la mayoría de los ejemplos, actividades y problemas eran contextualizados, por lo tanto, relacionados con situaciones del mundo real y del propio entorno del alumnado" (Albiol, 2020, p. 22, traducción propia). Uno de los problemas con los que justifica su valoración es el relacionado con la pandemia por COVID-19, que se presenta en la Figura 4:

Figura 4. Tercer problema de contextualización (modelización) propuesto en el TFM

\section{Pendiente Coronavirus}

Una de las cosas para las que sirven las matemáticas es la posibilidad que nos da para razonar y tener un buen criterio para saber cómo son los hechos en el mundo real. Por ejemplo, analizaremos la trágica gráfica de defunciones debido a la pandemia por coronavirus durante los primeros 28 días. Nos fijaremos en un periodo de 7 días, concretamente, desde el día 21 hasta el 28, y analizaremos la pendiente, o sea, la relación que existe entre el número de muertos dividido por el número de días. Supondremos que las líneas son prácticamente rectas, tal y como están remarcados en negro Italia y España en la gráfica.

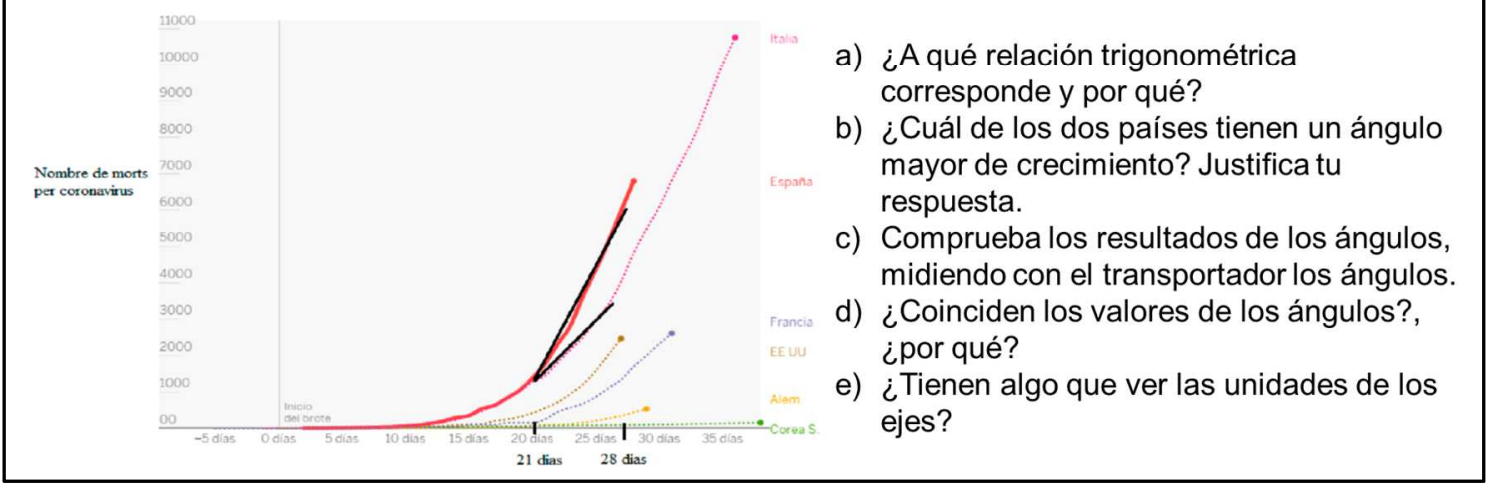

Fuente: Traducido desde Albiol (2020, pp. 69-70)

Finalmente, en el componente 'innovación didáctica', el docente en formación destaca que en su propuesta se introducen conceptos nuevos para sus estudiantes (razones trigonométricas, concepto de radián) y que, dado el contexto del trabajo telemático, el uso de recursos tecnológicos (PowerPoint, Excel, Google Classroom, Thatquiz, Socrative, etc.) para la resolución de actividades, representó una innovación en su práctica docente. 


\subsection{Valoración global de la idoneidad didáctica}

En la Figura 5 se presenta la valoración global que hace el futuro profesor Albiol (2020) sobre la idoneidad didáctica del proceso de instrucción implementado, a través del modelo que se explicó a los futuros docentes durante su ciclo formativo en el máster. Éste consiste en un gráfico radial de forma hexagonal, en el que el hexágono regular exterior representa un proceso de instrucción ideal, y el hexágono irregular interior representa la idoneidad del proceso de instrucción implementado. De este modo, el profesor en formación construyó el gráfico (ver Figura 5) basado en las valoraciones asignadas a cada idoneidad, tal como se mencionó en los subapartados anteriores.

Figura 5. Hexágono de valoración de las idoneidades

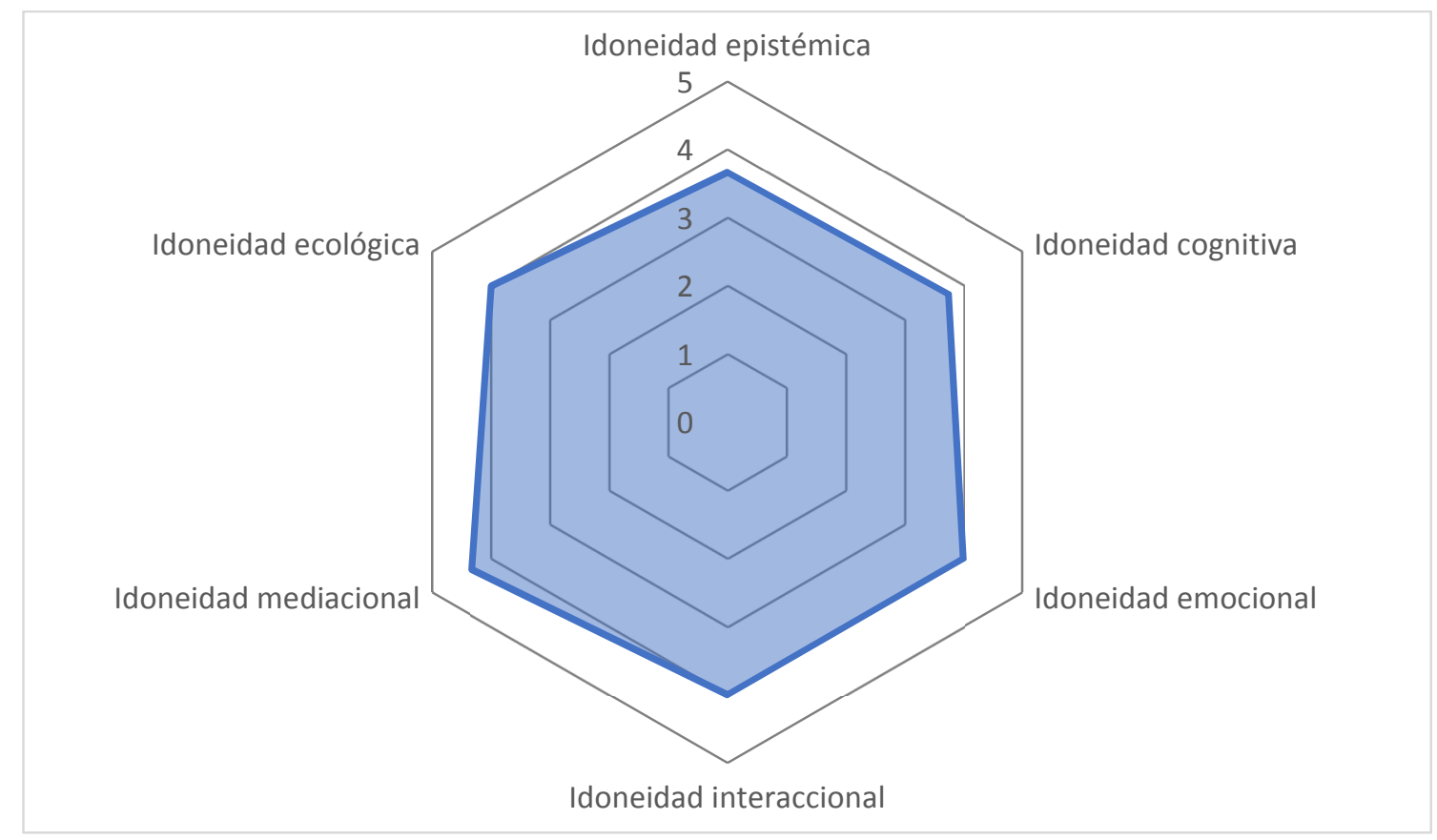

Fuente: Adaptado desde Albiol (2020, p. 23)

\subsection{Propuesta de mejora}

Con el fin de rediseñar su propuesta, el futuro profesor plantea una serie de elementos que considera como mejoras significativas, tanto en lo que respecta a la planificación de su unidad didáctica, como a la formulación de nuevas actividades, ello a partir de las valoraciones presentadas en los subapartados anteriores. En cuanto a la idoneidad epistémica, tal como se describió en el subapartado sobre esta valoración, el profesor en formación propone la mejora del componente 'representatividad de la complejidad', a través de la ampliación de los significados parciales de los objetos teorema de Thales, teorema de Pitágoras, y razones 
trigonométricas (seno, coseno y tangente). Junto con lo anterior, plantea algunas actividades adicionales que implican el uso del software GeoGebra y de material manipulativo. Un problema que destacar entre los nuevos que propone es el que se presenta en la Figura 6.

Figura 6. Problema de 'contextualización (modelización)' propuesto en el rediseño

\section{Pasillos móviles}

¿Sabemos cómo desplazarnos con el COVID?

Un fabricante de pasillos móviles está ideando un nuevo modelo a raíz de las nuevas medidas de distanciamiento en lugares muy transitados, como grandes avenidas, aeropuertos, estaciones de trenes, centros comerciales, accesos a estadios deportivos, conciertos musicales, espectáculos, etc.

Imagínense que necesitan hacer el máximo número de marcas sobre la plataforma móvil para que los peatones no se tengan que mover y así mantener la distancia de seguridad.
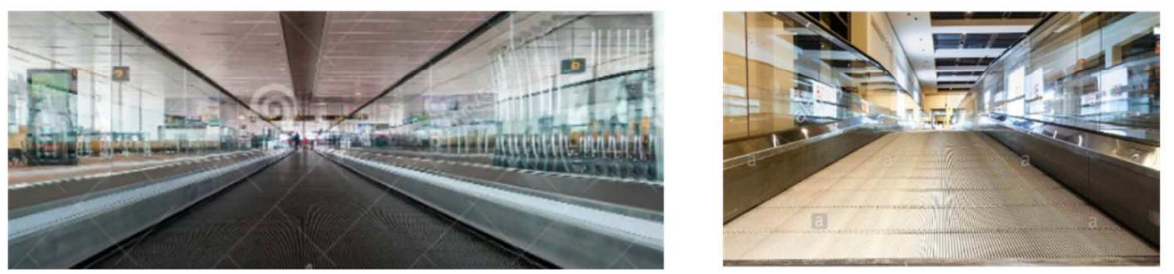

a) ¿Cómo lo diseñarías?, ¿qué anchura de la plataforma sería la ideal?, ¿cuántas posibilidades hay?

b) ¿Se parece a alguna forma geométrica?

c) En caso afirmativo, ¿cuántas personas forman esta forma geométrica?

d) ¿Qué distancia hay entre dos personas y qué ángulos hay entre tres personas de esta forma geométrica? Encuentra todas las posibilidades.

Fuente: Traducido desde Albiol (2020, p. 70-71)

En palabras de su autor, "con esta actividad se pretende que los alumnos experimenten con situaciones cercanas y realistas, y hagan uso de la trigonometría para aspectos de mucha utilidad" (Albiol, 2020, p. 70, traducción propia). El problema lo plantea para reforzar el proceso de 'contextualización (modelización)', en la reformulación de la quinta sesión de su unidad didáctica, cuyo contenido a abordar es la relación entre geometría y trigonometría.

Entre las otras propuestas de rediseño que plantea el docente en formación, se encuentran la mejora de la consolidación y reforzamiento de los conocimientos previos para los teoremas de Thales y Pitágoras, ello a través del uso de material manipulativo; el reajuste de los contenidos y actividades para cada sesión, a fin de optimizar los tiempos de implementación; etc. No obstante, un elemento que mantiene es la metodología de trabajo grupal entre los estudiantes (grupos de 2 o 3 alumnos), sobre todo en lo que respecta a la resolución de problemas, ello justificado en lo planteado por Coll y Colomina (1990) acerca del desarrollo de procesos de construcción compartidos con influencia educativa de los compañeros de aula (funciones de regulación y desarrollo emocional). 


\section{Discusión y conclusiones}

Del análisis de la reflexión realizada por el futuro profesor Albiol (2020), los resultados evidencian que el grueso de las valoraciones sobre la incorporación de la modelización en su unidad didáctica se centra en los criterios de idoneidad epistémica y ecológica.

Con respecto a la idoneidad epistémica, dentro del componente 'riqueza de procesos', el profesor en formación define los procesos 'resolución de problemas' y 'contextualización (modelización)', pero no indica un referente concreto en el que se basó para tales definiciones.

Sobre el proceso 'resolución de problemas', la definición que propone el currículo de la ESO es la siguiente:

Un problema es una propuesta de enfrentamiento con una situación desconocida que se plantea a través de un conjunto de datos dentro de un contexto, para la que, en principio, no se dispone de una respuesta inmediata y que requiere reflexionar, tomar decisiones y diseñar estrategias. (Departament d'Educació, 2019, p. 141, traducción propia).

No obstante, el futuro docente se refiere indistintamente a las actividades que propone como ejercicios o problemas, a lo largo de su memoria escrita.

Sobre el proceso 'contextualización (modelización)', el autor del TFM utiliza indistintamente los términos 'contextualización' y 'modelización', refiriéndose a los mismos como un único proceso. Una posible causa de esta identificación es que el docente en formación no tenga una claridad conceptual sobre la teorización en torno a la modelización matemática, lo que puede justificar que no haga una separación y distinción entre ambos términos. Del mismo modo, el análisis de contenido permitió evidenciar que el autor del TFM asume la perspectiva de 'modelización como un vehículo', en que a través de la resolución de problemas con un contexto que incluye elementos del mundo real, pretende que los estudiantes construyan y refuercen los contenidos asociados a la trigonometría. Esta postura, además, guarda relación con la estructura del sistema educativo en que se encuentra inserta esta propuesta, donde la modelización se considera como un complemento para el aprendizaje de los estudiantes sobre un contenido matemático (en este caso, la trigonometría), y no como la competencia a desarrollar en los estudiantes, supeditando de este modo la modelización al contenido matemático. A partir de esto último, se desprende la idea de que, aun cuando un profesor tenga un conocimiento teórico-conceptual sobre modelización, se puede encontrar con restricciones 
de tiempos curriculares para desarrollar más ampliamente esta competencia en sus estudiantes y, probablemente, sólo pueda plantear problemas de consolidación hacia el final de un tema o unidad específicos que incluyan las características que plantea Maßß (2010) para un 'problema de modelización' (descritas en el subapartado 2.1).

Con todo lo mencionado anteriormente, se hace evidente que el TFM analizado contempla ejercicios y 'problemas verbales (word problems)', pero ningún 'problema de modelización' como tal. Más en concreto, las actividades mostradas en la Figura 2 y Figura 3 corresponden a ejercicios algorítmicos; y las mostradas en la Figura 4 y Figura 6, se condicen mayormente con lo definido por Verschaffel, Greer y de Corte (2000) como una descripción verbal de una situación-problema, en la que se plantean preguntas que pueden ser respondidas a través de la aplicación de operaciones matemáticas a los datos numéricos que se incluyen en el enunciado.

Con respecto a la idoneidad ecológica, dentro del componente 'utilidad socio-laboral', el futuro profesor destaca la importancia de haber trabajado el proceso de 'contextualización (modelización)' en el planteamiento de problemas en su unidad didáctica. En menor medida, dentro del componente 'conexiones intra e interdisciplinares', el profesor en formación hace alusión al planteamiento de actividades -consideradas por el autor como problemas de 'contextualización (modelización)' - para relacionar los contenidos matemáticos con otras asignaturas. Sin embargo, esta situación se acerca más al proceso de 'aplicación matemática' (matemáticas $\rightarrow$ realidad) para la resolución de problemas que al de 'modelización matemática', en términos de lo consensuado por Blum (1994, 2002).

Retomando la pregunta de investigación de este estudio, sobre cómo utilizan los profesores en formación los criterios de idoneidad didáctica en sus trabajos finales de máster para reflexionar sobre la implementación de la modelización, se puede afirmar que estos criterios no sólo permitieron al autor del TFM reflexionar sobre su práctica docente, sino también, desvelar tanto sus nociones conceptuales sobre la modelización como la importancia que le atribuye a la misma dentro del proceso de instrucción implementado. Todo ello, a través de las valoraciones realizadas en los criterios y componentes antes mencionados. A modo de conclusión sobre el propio aprendizaje en el máster, y aludiendo claramente a los criterios de idoneidad didáctica, el futuro profesor declara lo siguiente: 
Gracias a la diversidad de disciplinas que hemos estudiado, soy consciente de la importancia que tiene como profesor dominar los contenidos desde múltiples representaciones, pero a la vez, ser paciente y planificado para optimizar el aprendizaje de nuestros alumnos en todos los sentidos (epistemológico, emocional, cognitivo, interaccional, mediacional, y ecológico). (Albiol, 2020, p. 30, traducción propia).

Otra conclusión a destacar es aquélla que alude a la influencia que tuvieron las asignaturas del módulo específico en la reflexión sobre su propia práctica:

Aunque considero positiva la implementación de la UD [Unidad Didáctica] que hice en el prácticum, muchos aspectos que se nos han presentado en Enseñanza y aprendizaje, en Innovación e investigación, y en Complementos de formación, me han hecho reflexionar sobre la riqueza de procesos y la calidad de enseñanza que tiene el aplicar la tendencia constructivista, debido a que son los propios alumnos los que construyen el conocimiento. Como docente, pienso que debería mejorar este aspecto. (Albiol, 2020, p. 30, traducción propia).

Una última conclusión que se pide a los estudiantes del máster es que realicen una autoevaluación competencial, de acuerdo con la propuesta de Font et al. (2012). Para ello, deben comparar el nivel de cada competencia, considerando el que tenían al ingresar al programa de máster con el que lograron desarrollar al culminar su proceso formativo. Sobre la competencia 9 (Contextualización y valor interdisciplinario), el futuro profesor afirma:

$\mathrm{N} 2 \rightarrow \mathrm{N} 3$. Ya era consciente de la importancia de las matemáticas en otros contextos y, sobre todo, con otras disciplinas como la física, la biología, la educación física, pero con el enfoque contextualizado de las nuevas tendencias en didáctica de las matemáticas, he aprendido a desarrollar esta competencia. (Albiol, 2020, p. 33, traducción propia).

Finalmente, este estudio forma parte de una investigación más amplia, en que, por una parte, se analiza la actividad matemática del proceso de modelización a través de la utilización de las herramientas aportadas por el EOS (véase Ledezma, Font y Sala, en prensa) y, por otra, 
se estudia la importancia que le atribuyen los profesores en formación a dicho proceso en sus propuestas didácticas, siguiendo la línea de este artículo.

\section{Agradecimientos}

Este estudio fue realizado en el marco del Proyecto ANID/PFCHA nro. 72200458 (Chile), y del Proyecto de Investigación en Formación de Profesorado PGC2018-098603-B-I00 (MCIU/AEI/FEDER, UE).

\section{Referencias}

Abassian, A., Safi, F., Bush, S. y Bostic, J. (2020). Five different perspectives on mathematical modeling in mathematics education. Investigations in Mathematics Learning, 12(1), 53-65. https://doi.org/10.1080/19477503.2019.1595360

Albiol, A. (2020). Trigonometria a Quart d'ESO: proposta de millora (Trabajo final de máster no publicado). Universitat de Barcelona, España.

Blum, W. (1994). Mathematical modelling in mathematics education and instruction. En T. Breiteig, I. Huntley y G. Kaiser-Messmer (Eds.), Teaching and Learning Mathematics in Context (pp. 3-14). Chichester, Inglaterra: Ellis Horwood.

Blum, W. (2002). ICMI Study 14: Applications and modelling in mathematics education Discussion document. Educational Studies in Mathematics, 51(1-2), 149-171. https://doi.org/10.1023/a:1022435827400

Blum, W. (2011). Can modelling be taught and learnt? Some answers from empirical research. En G. Kaiser, W. Blum, R. Borromeo Ferri y G. Stillman (Eds.), Trends in Teaching and Learning of Mathematical Modelling: ICTMA 14 (pp. 15-30). Dordrecht, Países Bajos: Springer. https://doi.org/10.1007/978-94-007-0910-2_3

Blum, W. y Leiß, D. (2007). How do students and teachers deal with modelling problems? En C. Haines, P. Galbraith, W. Blum y S. Khan (Eds.), Mathematical Modelling (ICTMA 12): Education, Engineering and Economics (pp. 222-231). Chichester, Inglaterra: Horwood. https://doi.org/10.1533/9780857099419.5.221

Borromeo Ferri, R. (2006). Theoretical and empirical differentiations of phases in the modelling process. Zentralblatt für Didaktik der Mathematik, 38(2), 86-95. https://doi.org/10.1007/bf02655883

Borromeo Ferri, R. (2018). Learning How to Teach Mathematical Modeling in School and Teacher Education. Cham, Suiza: Springer. https://doi.org/10.1007/978-3-319-68072-9

Breda, A. (2020). Características del análisis didáctico realizado por profesores para justificar la mejora en la enseñanza de las matemáticas. BOLEMA: Boletim de Educação Matemática, 34(66), 69-88. https://doi.org/10.1590/1980-4415v34n66a04

Breda, A., Font, V., Lima, V. M. R. y Villela, M. (2018). Componentes e indicadores de los criterios de idoneidad didáctica desde la perspectiva del enfoque ontosemiótico. Transformación, 14(2), 162-176. 
Breda, A. y Lima, V. M. R. (2016). Estudio de caso sobre el análisis didáctico realizado en un trabajo final de un máster para profesores de matemáticas en servicio. REDIMAT: Journal of Research in Mathematics Education, 5(1), 74-103. https://doi.org/10.4471/redimat.2016.1955

Breda, A., Pino-Fan, L. y Font, V. (2017). Meta didactic-mathematical knowledge of teachers: Criteria for the reflection and assessment on teaching practice. EURASIA: Journal of Mathematics Science and Technology Education, 13(6), 1893-1918. https://doi.org/10.12973/eurasia.2017.01207a

Cohen, L., Manion, L. y Morrison, K. (2018). Research Methods in Education (8va ed.). Nueva York, NY: Routledge.

Coll, C. y Colomina, R. (1990). Interacción entre alumnos y aprendizaje escolar. En J. Palacios, Á. Marchesi y C-Coll (Eds.), Desarrollo Psicológico y Educación Vol. II: Psicología de la Educación (pp. 335-352). Madrid, España: Editorial Alianza.

Departament d'Educació. (2019). Currículum Educació Secundària Obligatòria. Barcelona, España: Generalitat de Catalunya.

Font, V., Breda, A., Seckel, M. J. y Pino-Fan, L. R. (2018). Análisis de las reflexiones y valoraciones de una futura profesora de matemáticas sobre la práctica docente. Ciencia y Tecnología, 43(2), 62-75.

Font, V., Giménez, J., Zorrilla, J. F., Larios, V., Dehesa, N., Aubanell, A. y Benseny, A. (2012). Competencias del profesor y competencias del profesor de matemáticas. Una propuesta. En V. Font, J. Giménez, V. Larios y J. F. Zorrilla (Eds.), Competencias del Profesor de Matemáticas de Secundaria y Bachillerato (pp. 59-68). Barcelona, España: Publicacions i Edicions de la Universitat de Barcelona.

Font, V., Godino, J. D. y Gallardo, J. (2013). The emergence of objects from mathematical practices. Educational Studies in Mathematics, 82(1), 97-124. https://doi.org/10.1007/s10649-012-9411-0

Godino, J. D. (2013). Indicadores de la idoneidad didáctica de procesos de enseñanza y aprendizaje de las matemáticas. Cuadernos de Investigación y Formación en Educación Matemática, 8(11), 111-132.

Godino, J. D., Batanero, C. y Font, V. (2007). The onto-semiotic approach to research in mathematics education. ZDM Mathematics Education, 39(1), 127-135. https://doi.org/10.1007/s11858-006-0004-1

Godino, J. D., Batanero, C. y Font, V. (2019). The Onto-Semiotic Approach: Implications for the prescriptive character of didactics. For the Learning of Mathematics, 39(1), 38-43.

Guerrero-Ortiz, C. y Mena-Lorca, J. (2015). Modelación en la enseñanza de las matemáticas: Matemáticos y profesores de matemáticas, sus estrategias. Revista Electrónica de Investigación en Educación en Ciencias, 10(1), 1-14.

Julie, C. y Mudaly, V. (2007). Mathematical modelling of social issues in school mathematics in South Africa. En W. Blum, P. L. Galbraith, H.-W. Henn y M. Niss (Eds.), Modelling and Applications in Mathematics Education: The 14th ICMI Study (pp. 503-510). Boston, MA: Springer. https://doi.org/10.1007/978-0-387-29822-1_58 
Ledezma, C., Font, V. y Sala, G. (en prensa). Mathematical modelling from a networking of theories between the modelling cycle from a cognitive perspective and the onto-semiotic approach. Educational Studies in Mathematics.

Lesh, R. y Doerr, H. M. (2003). Foundations of a models and modeling perspective on mathematics teaching, learning, and problem solving. En R. Lesh y H. M. Doerr (Eds.), Beyond Constructivism: Models and Modeling Perspectives on Mathematics Problem Solving, Learning, and Teaching (pp. 3-33). Mahwah, NJ: Lawrence Erlbaum.

Maaß, K. (2010). Classification scheme for modelling tasks. Journal für Mathematik-Didaktik, 31(2), 285-311. https://doi.org/10.1007/s13138-010-0010-2

Morales-López, Y. y Font, V. (2019). Valoración realizada por una profesora de la idoneidad de su clase de matemáticas. Educação e Pesquisa, 45, e189468.

Palm, T. (2007). Features and impact of the authenticity of applied mathematical school tasks. En W. Blum, P. L. Galbraith, H.-W. Henn y M. Niss (Eds.), Modelling and Applications in Mathematics Education: The 14th ICMI Study (pp. 201-208). Boston, MA: Springer. https://doi.org/10.1007/978-0-387-29822-1 20

Pino-Fan, L., Godino, J. D. y Font, V. (2016). Assessing key epistemic features of didacticmathematical knowledge of prospective teachers: The case of the derivative. Journal of Mathematics Teacher Education, 21(1), 63-94. https://doi.org/10.1007/s10857-016-9349-8

Sala, G., Font, V., Giménez, J. y Barquero, B. (2017). Inquiry and modelling in a real archaeological context. En G. Stillman, W. Blum y G. Kaiser (Eds.), Mathematical Modelling and Applications: Crossing and Researching Boundaries in Mathematics Education (pp. 325-335). Cham, Suiza: Springer. https://doi.org/10.1007/978-3-319-62968128

Schoenfeld, A. H. (Ed.). (1994). Mathematical Thinking and Problem Solving. Hillsdale, MI: Erlbaum.

Schoenfeld, A. H. y Kilpatrick, J. (2008). Towards a theory of proficiency in teaching mathematics. En D. Tirosh y T. Woods (Eds.), The International Handbook of Mathematics Teacher Education Vol. 2: Tools and Processes in Mathematics Teacher Education (pp. 321-354). Róterdam, Países Bajos: Sense Publishers.

Schön, D. A. (1983). The Reflective Practitioner: How Professionals Think in Action. Nueva York, NY: Basic Books.

Schön, D. A. (1987). Educating the Reflective Practitioner: Towards a New Design for Teaching and Learning in the Professions. San Francisco, CA: Jossey-Bass Publishers.

Stake, R. E. (2005). Qualitative case studies. En N. K. Denzin y Y. S. Lincoln (Eds.), The SAGE Handbook of Qualitative Research (3ra ed.) (pp. 443-466). Thousand Oaks, CA: Sage.

Verschaffel, L., Greer, B. y de Corte, E. (2000). Making Sense of Word Problems. Lisse, Países Bajos: Swets \& Zeitlinger. 